\title{
An Overview of the Obstacle Problem
}

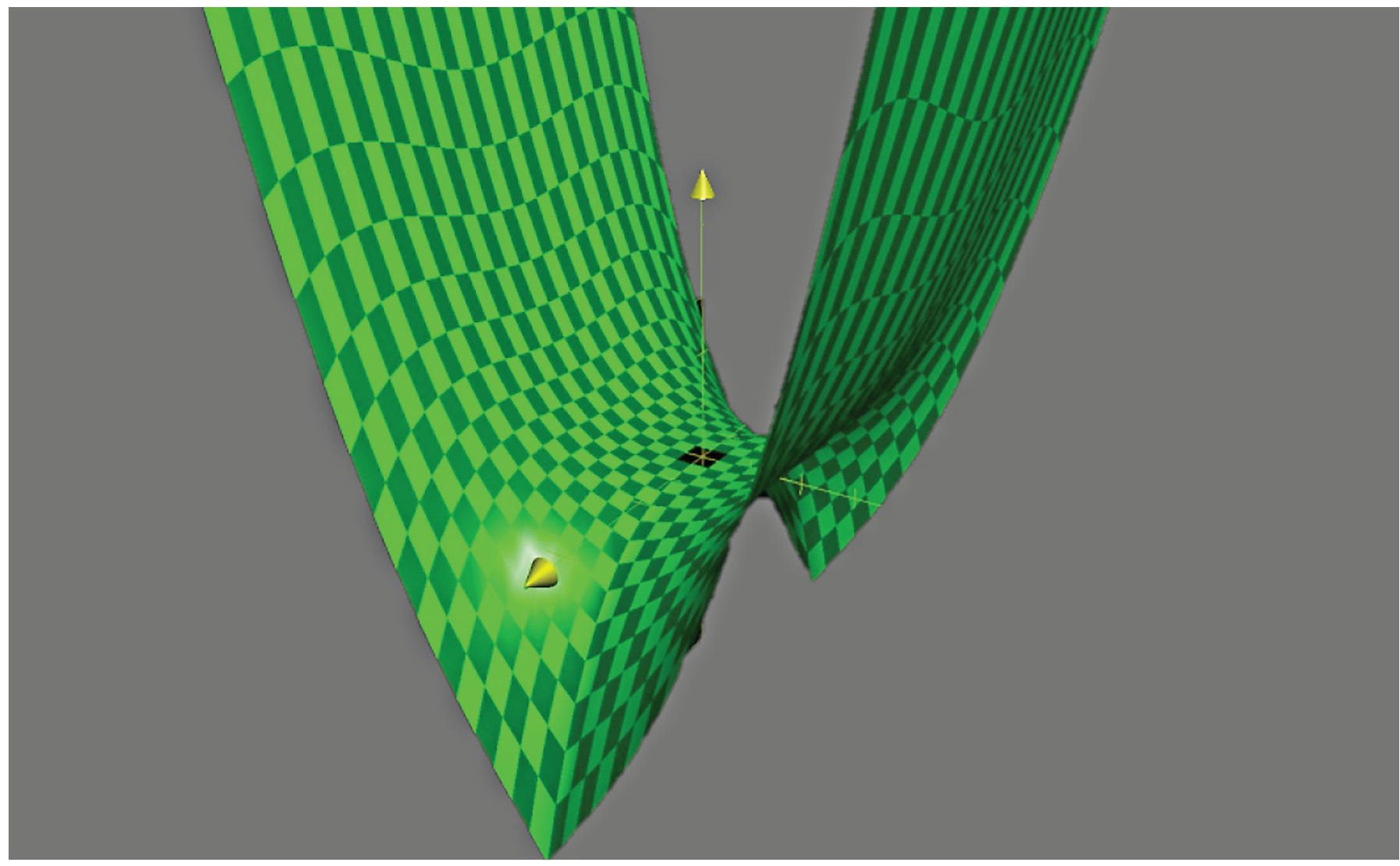

Donatella Danielli

\section{Introduction}

Free Boundary Problems naturally occur in physics and engineering when a conserved quantity or relation changes discontinuously across some value of the variables under consideration. In mathematical terms, they consist in solving partial differential equations (PDEs) in a domain, a part of whose boundary is a priori unknown, and which has to be determined as part of the problem. Said portion of the boundary is called the free boundary, and in models it appears, for instance, as the interface between a fluid and

Donatella Danielli is a professor of mathematics at Purdue University. Her email address is danie11i@math.purdue.edu.

Communicated by Notices Associate Editor Daniela De Silva.

For permission to reprint this article, please contact:

reprint-permission@ams.org. air, or water and ice. In addition to the usually prescribed initial and boundary values, other conditions, arising from the physical laws governing the model, are imposed at the free boundary. Since in general, with few exceptions, it is impossible to explicitly determine the solution and the underlying domain, the ultimate goal consists in establishing analytic and geometric properties for both. In the past few decades this area of research has seen deep and broad advancements, due to the assimilation of problems coming from other applied sciences, such as finance, mathematical biology, and population dynamics, just to name a few. Its development has been intrinsically intertwined with the theory of Variational Inequalities, born in Italy in the early 1960s. Its founding fathers were Guido Stampacchia, who was motivated by questions in potential theory, and Gaetano Fichera, who instead was interested in problems in elasticity with unilateral constraints (more on this later...). 


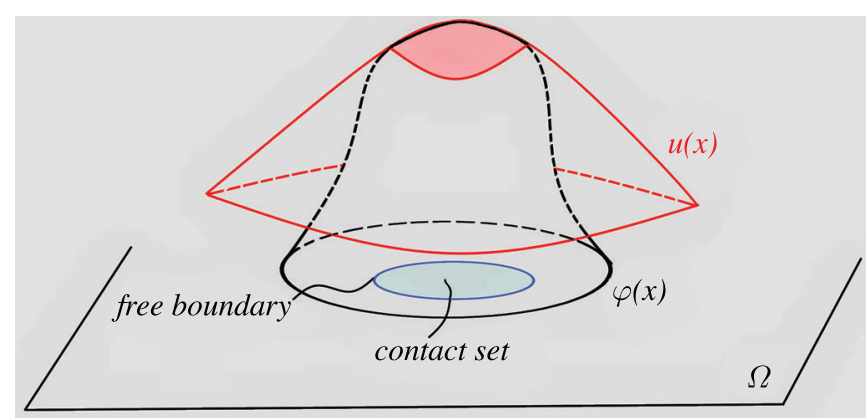

Figure 1. Example of an obstacle problem.

Over the years, Variational Inequalities have become an essential tool in various sectors of applied mathematics. From a more theoretical standpoint, they appear in the Calculus of Variations when a function is minimized over a set of constraints, giving rise to a set of differential inequalities which replace the classical Euler-Lagrange equation. Free Boundary Problems and Variational Inequalities have generated new and exciting ideas based on the interplay of methods from PDEs, the Calculus of Variations, Geometric Measure Theory, and Mathematical Modeling. One of the main driving forces behind the development of their theory has been the study of the obstacle problem.

\section{The Classical Theory}

In its classical formulation, the obstacle problem consists in finding the equilibrium configuration of an elastic membrane whose boundary is held fixed, and which is constrained to lie above a given obstacle. Mathematically, we seek to minimize the Dirichlet energy

$$
J(v)=\int_{\Omega}|\nabla v|^{2}
$$

in a domain $\Omega \subset \mathbb{R}^{n}$, among all configurations $v(x)$ (representing the vertical displacement of the membrane) with prescribed boundary values $\left.v\right|_{\partial \Omega}=f(x)$, and constrained to remain above the obstacle $\varphi(x)$, that is, in the class

$$
\mathcal{K}=\left\{v \in W^{1,2}(\Omega)|v|_{\partial \Omega}=f(x), v \geq \varphi\right\},
$$

given the compatibility condition $\varphi \leq f$ on $\partial \Omega$. We will denote by $u$ the solution of this minimization problem. The domain $\Omega$ then breaks down into a region where $u$ coincides with the obstacle function, known as the contact set, and a region where the solution is above the obstacle. The free boundary is defined as the topological boundary of the contact set, that is,

$$
\mathcal{F}(u)=\partial\{x \in \Omega \mid u(x)=\varphi(x)\} .
$$

We explicitly observe that, as it often happens in the Calculus of Variations, searching for solutions in an apparently natural but too narrow family of admissible functions may produce no results. This justifies the choice of the class of competitors as the Sobolev space $W^{1,2}(\Omega)$, endowed with the inner product $\langle u, v\rangle=\int_{\Omega}(u v+\nabla u \cdot \nabla v) d x$. Since the functional $J(v)$ is continuous and strictly convex on the convex set $\mathcal{K}$, the existence and uniqueness of minimizers are guaranteed.

The obstacle problem can be reformulated as a Variational Inequality on $W^{1,2}(\Omega)$. In fact, solving the obstacle problem is equivalent to determining a function $u \in \mathcal{K}$ such that

$$
\int_{\Omega} \nabla u \cdot \nabla(v-u) d x \geq 0 \quad \text { for all } v \in \mathcal{K} .
$$

Standard variational arguments show that the solution is harmonic away from the contact set, or

$$
\Delta u=0 \quad \text { in }\{u>\phi\},
$$

and superharmonic on the contact set, i.e.,

$$
\Delta u \leq 0 \quad \text { in }\{u=\phi\} .
$$

Hence, the solution is a superharmonic function in $\Omega$, in the sense of distributions. Based on these considerations, we can rewrite the obstacle problem in yet another way, namely as

$$
\min \{-\Delta u, u-\varphi\}=0,
$$

subject to the boundary condition $\left.u\right|_{\partial \Omega}=f(x)$.

Two natural questions arise at this point:

1. How regular is the function $u$ ?

2. What are the geometric properties of the contact set? Is the free boundary a smooth surface?

Concerning the first question, it was shown in [Fre72] that the solution is in $C^{1,1}(\Omega)$ (assuming that $\varphi$ is at least in the same class), i.e., it has bounded second derivatives. It is readily verified that such regularity is optimal. Heuristically, $\Delta u$ jumps from 0 on the set where $u$ is detached from $\varphi$, to $\Delta \varphi$ on the contact set, and therefore it is unreasonable to expect continuity of the second derivatives.

The second question is significantly more delicate and complex. Before we begin to outline the key steps in its answer, let us mention that from the point of view of applications, smoothness properties of the interface are crucial to develop, for instance, robust numerical methods. The first fundamental accomplishment in this direction is due to D. Kinderlehrer and L. Nirenberg [KN77], who showed that $C^{1}$ free boundaries are, in fact, analytic. Applicability of this result, however, rests on the initial knowledge of a certain degree of smoothness, whereas a priori the interface could be a very irregular object. The breakthrough came from the seminal paper [Caf77], where L. Caffarelli, inspired by De Giorgi's approach for the regularity of areaminimizing surfaces, introduced one of the most transformative ideas in the theory of free boundary problems, namely the use of blow-up arguments. In layman's terms, they consist in zooming in on a fixed free boundary point, as if using a magnifying glass, and observing the properties 
of the free boundary at a very large scale. The mathematical tool corresponding to the magnifying glass is the rescaling (see Figure 2). To illustrate its workings, we follow here

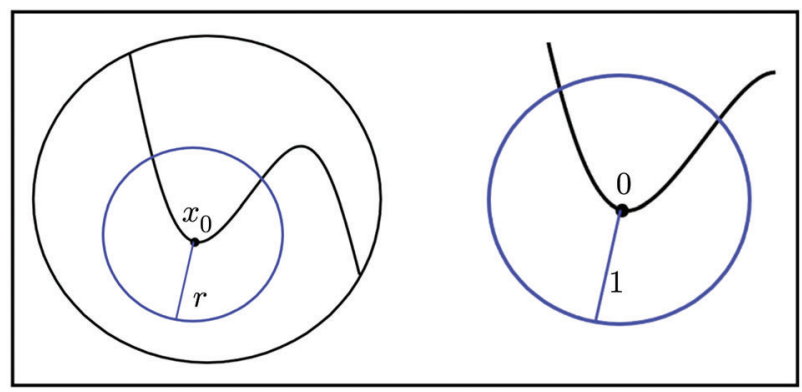

Figure 2. The effect of rescaling.

the approach in [Caf98]. First of all, we reduce the problem to a zero-obstacle one by replacing $u$ with $w=u-\varphi$. Then, in the region $\{w>0\}$, one has

$$
\Delta w=\Delta(u-\varphi)=-\Delta \varphi:=g(x) .
$$

For the sake of simplicity we will assume $g(x) \equiv 1$, and continue to denote the solution to this normalized problem by $u$. The starting point is the observation that $u$ exhibits quadratic growth near a free boundary point $x_{0} \in \mathcal{F}(u)$. More precisely, there exist two constants $c, C>0$ such that

$$
c r^{2} \leq \sup _{B_{r}\left(x_{0}\right)} u \leq C r^{2},
$$

where $B_{r}\left(x_{0}\right)=\left\{x \in \mathbb{R}^{n}|| x-x_{0} \mid<r\right\}$. This estimate suggests the following family of rescaled functions:

$$
u_{r}(x)=\frac{1}{r^{2}} u\left(x_{0}+r x\right)
$$

for $r>0$. The boundedness of the second derivatives of $u$ allows one to apply the classical compactness result of Ascoli-Arzelà. We thus infer that, possibly passing to a subsequence $r_{j} \rightarrow 0^{+}, u_{r}(x)$ converges to a function $u^{*}(x)$, called a blow-up limit (or simply blow-up) relative to $x_{0} \in \mathcal{F}(u)$. The following classification of blow-ups holds:

(i) either $u^{*}$ is a half-space solution to the obstacle problem of the form

$$
u^{*}(x)=\frac{1}{2} \max \{x \cdot e, 0\}^{2}
$$

for some $e$ unit vector in $\mathbb{R}^{n}$,

(ii) or $u^{*}$ is a parabola solution of the form

$$
u^{*}(x)=\frac{1}{2} x \cdot A x
$$

for some matrix $A$, with $A$ nonnegative definite and $\operatorname{trace}(A)=1$.

This alternative leads to a corresponding characterization of the free boundary point $x_{0}$. If (i) holds, we say that $x_{0}$ is a regular free boundary point, and otherwise we say that $x_{0}$ is singular; see Figure 3 .
As one may surmise from the nomenclature, the objective is now to show that the free boundary can be expressed as a smooth hypersurface in a neighborhood of a regular point, whereas smoothness is not expected around a singular one. Cusp-like or pinched bottleneck singularities, in fact, may occur even when the obstacle is smooth. The main difficulty in the study of the free boundary at this point lies in transferring the information acquired on the shape of the blow-ups back to the original configuration. In principle, in fact, a point $x_{0} \in \mathcal{F}(u)$ could be regular and singular at the same time, depending on the choice of the subsequence $r_{j}$ taken to pass to the limit. Geometric considerations, however, prove that this is not possible.

In case (i), to fix ideas assume $u^{*}(x)=\frac{1}{2}\left(x_{n}^{+}\right)^{2}$. The new contact set $\left\{u^{*}=0\right\}$ is the half-space $\left\{x_{n} \leq 0\right\}$ and therefore, undoing the rescaling, one can see that the original contact set $\{u=0\}$ has positive Lebesgue density at $x_{0}$, that is,

$$
\limsup _{r \rightarrow 0^{+}} \frac{\left|B_{r}\left(x_{0}\right) \cap\{u=0\}\right|}{r^{n}}>0 .
$$

This is a very stable situation, in the sense that the flatness of the free boundary for $u^{*}$ (namely the hyperplane $\left\{x_{n}=\right.$ $0\})$ translates into "almost-flatness" of the free boundary of the rescaled functions $u_{r}$ for $r$ sufficiently small, and thus for $u$ at a small scale. More precisely, for $\sigma>0$ and $0<r<r_{\sigma}$ it holds that

$$
\mathcal{F}\left(u_{r}\right) \cap B_{1 / 2}\left(x_{0}\right) \subset\left\{\left|x_{n}\right|<\sigma\right\} .
$$

The first step in establishing the smoothness of the free boundary is to prove its Lipschitz regularity. This easily follows from the fact that the directional derivatives $\partial_{e} u$ are nonnegative near a regular point for $e$ in a cone of directions

$$
C_{\delta}=\left\{x \in \mathbb{R}^{n}\left|x_{n}>\delta\right| x^{\prime} \mid\right\},
$$

with $x^{\prime}=\left(x_{1}, \ldots, x_{n-1}\right)$ and $0<\delta<1$; see Figure 4 . Without going into the technical details, the guiding principle here is that this monotonicity property, which clearly holds true for $u^{*}$, can be transferred to $u_{r}$ (for $r$

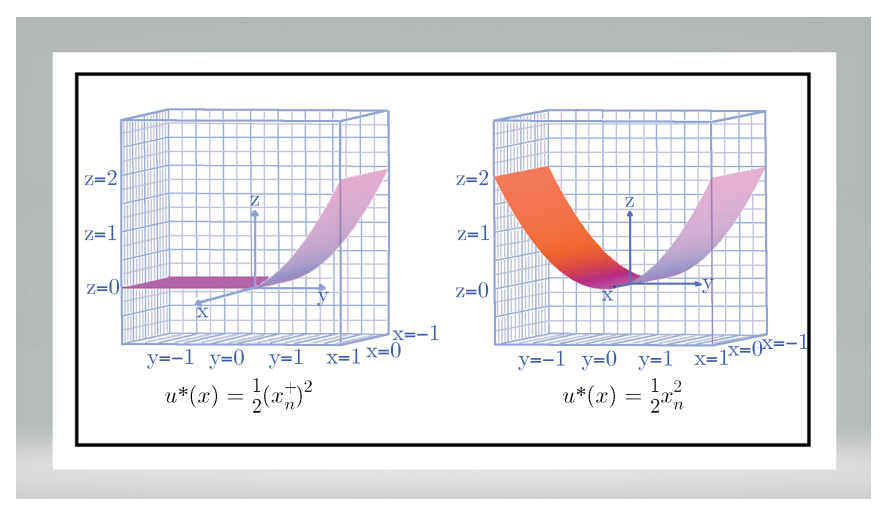

Figure 3. Blow-up limits at regular (left) and singular (right) free boundary points. 
small enough) combining the convergence in $C^{1}$ with (5), and then, "undoing" the rescaling, back to $u$ itself.

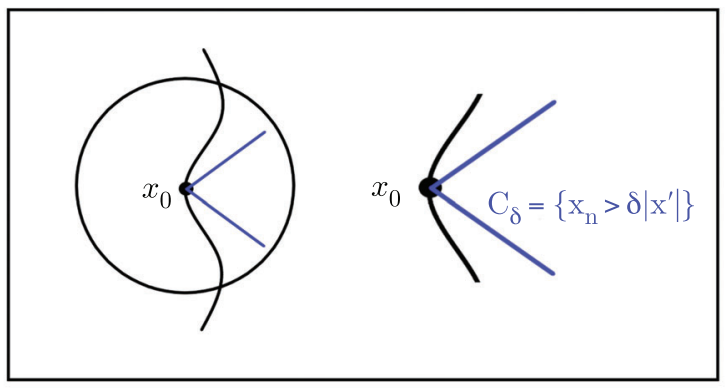

Figure 4. The cone of monotonicity.

Another relatively simple application of the directional monotonicity property yields that $\mathcal{F}(u)$ is locally a $C^{1}$ graph and this, combined with the result in [KN77], allows one to conclude that the free boundary is an analytic hypersurface in a neighborhood of a regular point.

We now turn our attention to the case

$$
\limsup _{r \rightarrow 0^{+}} \frac{\left|B_{r}\left(x_{0}\right) \cap\{u=0\}\right|}{r^{n}}=0 .
$$

Then it can be shown that $x_{0}$ is singular, and it holds that for any $r>0$ there exists a unit vector $e$ such that

$$
\mathcal{F}(u) \cap B_{r}\left(x_{0}\right) \subset\left\{x \in \mathbb{R}^{n}|| e \cdot\left(x-x_{0}\right) \mid \leq o(r)\right\} .
$$

What we have just described is the essence of the dichotomy theorem in [Caf77]. While the first part, relative to case (i), is definitive, the second one is not. In principle, in fact, the vector $e$ could depend, once more, on the subsequence chosen in the limiting process. In order to describe the structure of the free boundary near singular points, it is then pivotal to establish the uniqueness of the blowups. This is where monotonicity formulas enter the scene. In [Caf98], the uniqueness in the limiting process is accomplished by applying the celebrated Alt-Caffarelli-Friedman monotonicity formula to the first derivatives of the solution. A few years later, R. Monneau devised in [Mon03] an alternative approach (which does not require to differentiate the solution), based on a monotonicity formula that nowadays bears his name: if $x_{0}$ is a singular point, and $u^{*}=u_{x_{0}}^{*}$ is as in (4), then the functional

$$
\mathcal{M}\left(r ; u, u^{*}\right)=\int_{\partial B_{r}\left(x_{0}\right)}\left(u-u^{*}\right)^{2}
$$

is nondecreasing in $r>0$. This important property is deduced, in turn, from the nondecreasing character of the

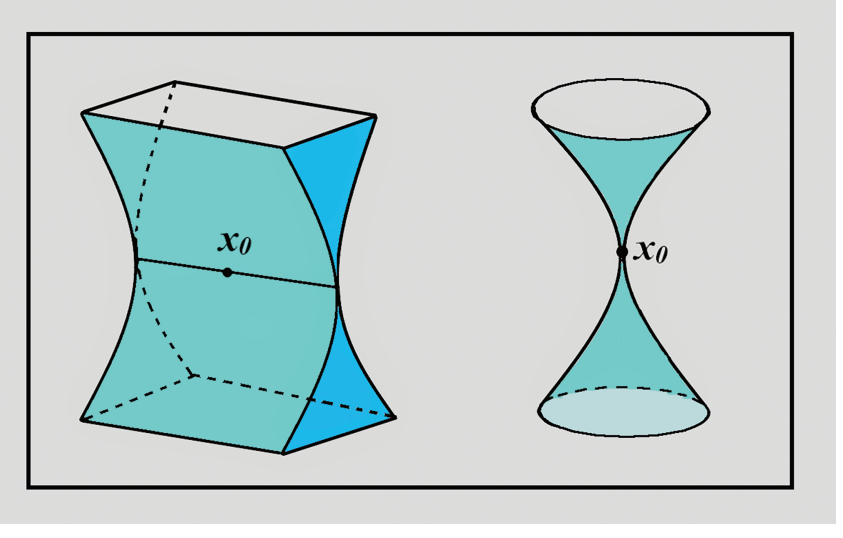

Figure 5. The different nature of singular points.

functional

$$
\begin{aligned}
\mathcal{W}(r ; u)= & \frac{1}{r^{n+2}} \int_{B_{r}\left(x_{0}\right)}\left(|\nabla u|^{2}+2 u\right) \\
& -\frac{1}{r^{n+3}} \int_{\partial B_{r}\left(x_{0}\right)} 2 u^{2},
\end{aligned}
$$

discovered by G. Weiss in [Wei99]. From Monneau's result one infers, in fact, not only uniqueness of the blow-up, but also the continuous dependence from the singular point: if we denote by $\Sigma(u)$ the collection of singular points, the map $\Sigma(u) \ni x_{0} \mapsto u_{x_{0}}^{*}$ is locally uniformly continuous. With this information at our disposal, it is finally possible to describe the set $\Sigma(u)$. We begin by observing that the nature of the free boundary can vary at different singular points, depending on the dimension $d_{x_{0}}$ of the set $\left\{u_{x_{0}}^{*}=\right.$ $0\}$. For instance, in the case $n=3$, if $u_{x_{0}}^{*}=\frac{1}{2} x_{1}^{2}$, then the contact set is asymptotically close to a plane, whereas it is asymptotically close to a line if $u_{x_{0}}^{*}=\frac{1}{2}\left(x_{1}^{2}+x_{2}^{2}\right)$; see Figure 5. It is thus natural to consider a classification of points in $\Sigma(u)$ based on $d_{x_{0}}$. More precisely, for $d=1, \ldots, n-1$, we introduce the set

$$
\Sigma_{d}=\left\{x_{0} \in \Sigma(u) \mid d_{x_{0}}=d\right\} .
$$

Caffarelli established in [Caf98] the first decisive result concerning the structure of the singular set. Namely, he proved that $\Sigma_{d}$ is locally contained in a $d$-dimensional manifold of class $C^{1}$. We illustrate here the main ideas of the proof. Note that, since $u \geq 0$ in $\Omega$ and $u=0$ on $\mathcal{F}(u)$, necessarily $\nabla u=0$ on $\mathcal{F}(u)$. In particular, $u=\nabla u=0$ in $\Sigma_{d}$ and thus, keeping in mind (3) and (4), we infer that $u_{x_{0}}^{*}$ corresponds, at least in principle, to the second-order term in the Taylor expansion of $u$ at $x_{0}$. Thanks to the continuity of the map $\Sigma(u) \ni x_{0} \mapsto u_{x_{0}}^{*}$, it is possible to apply Whitney's extension theorem to find a function $F: \mathbb{R}^{n} \rightarrow \mathbb{R}^{n}$ of class $C^{1}$ such that

$$
F\left(x_{0}\right)=\nabla u_{x_{0}}^{*}=0 \text { and } \nabla F\left(x_{0}\right)=D^{2} u_{x_{0}}^{*}
$$


for all $x_{0} \in \Sigma_{d}$. Invoking the definition of $\Sigma_{d}$, we have

$$
\operatorname{dim} \operatorname{ker} \nabla F\left(x_{0}\right)=\operatorname{dim} \operatorname{ker} D^{2} u_{x_{0}}^{*}=d
$$

in $\Sigma_{d}$. Applying the Implicit Function Theorem we can finally conclude that

$$
\Sigma_{d}=\{F=0\} \cap \Sigma_{d}
$$

is locally contained in a $C^{1} d$-dimensional manifold. When $n=2$, Weiss showed in [Wei99] that $C^{1}$-regularity can be improved to $C^{1, \alpha}$ by means of an epiperimetric-type approach, thus recovering an earlier result of L. Caffarelli and N. Rivière. Very recently, A. Figalli and J. Serra obtained in [FS19] an exhaustive characterization of the singular set: in two dimensions, $\Sigma(u)$ is locally contained in a $C^{2}$ curve, whereas in dimensions $n \geq 3$ singular points (except anomalous ones) can be covered by $C^{1,1}$-and in some cases $C^{2}$-manifolds. Additionally, they showed that the highest order stratum $\Sigma_{n-1}$ is always contained in a $C^{1, \alpha}$ manifold, thus generalizing Weiss's results. It should be noted that O. Savin and H. Yu have established in [SY19] essentially the same result for the obstacle problem associated to a convex fully nonlinear elliptic operator. They developed a novel method, based not on monotonicity formulas but on an iterative scheme, which provides an insightful alternative also for the classical problem.

\section{Thin Obstacles}

A problem in linear elasticity, first proposed by A. Signorini [Sig59] and now bearing his name, has been one of the mainsprings of the study of Variational Inequalities. In its simplest formulation, it consists of determining the displacements in a heavy, linearly elastic body resting on a rigid, frictionless horizontal plane (see Figure 6).

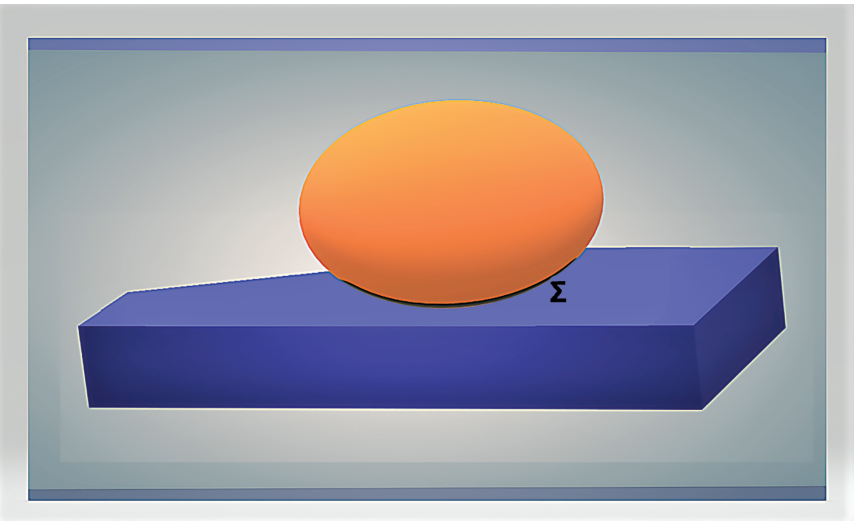

Figure 6. The classical Signorini problem.

The quintessential difficulty is the fact that the region of contact between the body and the plane is not known a priori, and it is thus part of the problem itself. The same mathematical model finds applications, e.g., in the study of semipermeable media, in problems of temperature control, and in financial mathematics. Since the first instance is the simplest to describe, we will derive the mathematical formulation of the problem from it. Semipermeable membranes allow the passage of fluid freely in one direction, but prevent all outflow of fluid in the other. They are used, for instance, in the process of hyperfiltration, or reverse osmosis. This is a pressure-driven mechanism to remove low molecular weight solutes, e.g., microorganic salts or small organic molecules (glucose, for example) from a solvent. In the natural process of osmosis, a membrane is used to separate two chambers of equal volume, for instance one filled with water and the other one with a dilute salt solution. Water is transferred from the solvent side of the membrane to the other chamber, until an osmotic equilibrium is reached. The hydrostatic pressure which has built up in the salt solution side of the membrane is known as osmotic pressure.

As the name implies, reverse osmosis is the reversal of this process. The application of a pressure, exceeding the osmotic one, on the salt solution in contact with the membrane forces water to flow from the chamber containing the salt solution to the other side (see Figure 7). The semipermeable nature of the membrane prevents the water from flowing back in, as would occur by natural osmosis. The water flux is proportional to the concentration difference across the membrane. This proportionality is expressed in terms of the permeability constant of the membrane, which in turn is inversely proportional to the membrane thickness.

Following [DL76], we now formulate the corresponding mathematical model. We let $\Omega$ be a region in $\mathbb{R}^{n}$ occupied by a viscous fluid which is only slightly compressible, with its pressure field denoted by $u(x)$. We assume that a smooth portion $\Gamma$ of $\partial \Omega$, with exterior unit normal $\nu$, consists of a semipermeable membrane of finite thickness. In correlation with the process of reverse osmosis, $\Omega$ represents the chamber containing the solvent (water), with $\Gamma$ separating $\Omega$ from another chamber filled with the dilute salt solution. Combining the law of conservation of mass

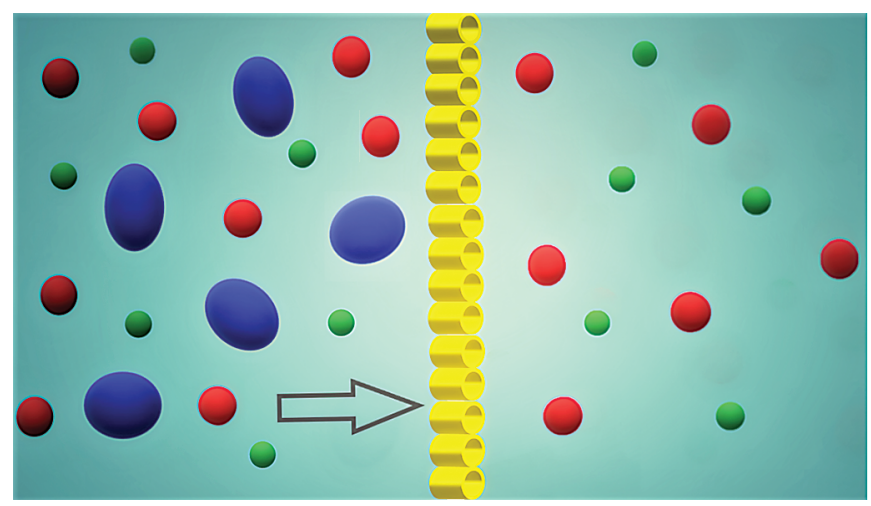

Figure 7. The process of reverse osmosis. 


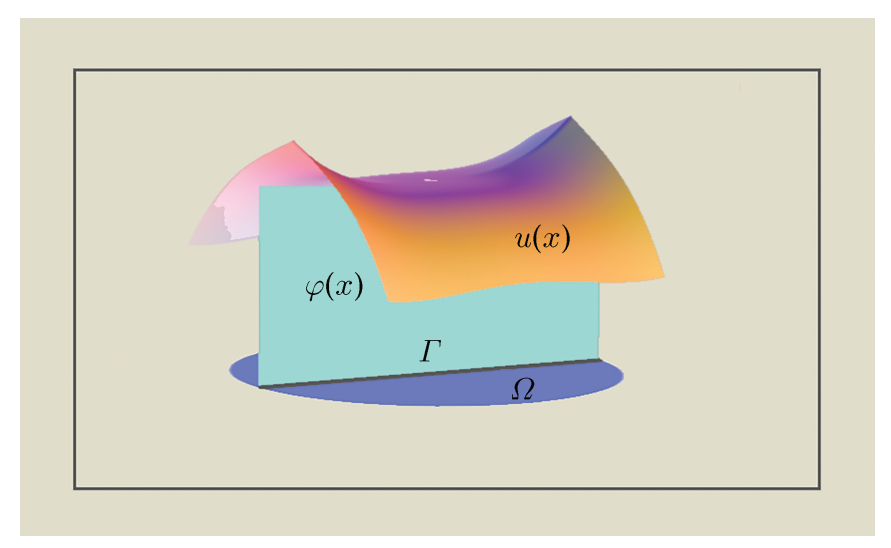

Figure 8. The thin obstacle problem.

with Darcy's law, one finds that the equilibrium configuration for $u$ satisfies the equation

$$
\Delta u=0 \text { in } \Omega .
$$

When a fluid pressure $\varphi(x)$ for $x \in \Gamma$ is applied on the outside of $\Omega$, one of two cases holds: $\varphi(x)<u(x)$, or $\varphi(x) \geq$ $u(x)$. In the former, the semipermeable wall prevents the fluid from leaving $\Omega$, so that the flux is null, or $\partial_{\nu} u=0$. In the latter case, instead, the fluid enters $\Omega$. Since the flow is proportional to the pressure differential, $\partial_{\nu} u=-\lambda(u-h)$, where $\lambda>0$ is the permeability constant of the membrane. Combining the two instances at once, we obtain the boundary condition $\partial_{\nu} u=\lambda(u-h)^{-}$. Given that the typical membrane thickness in the process of reverse osmosis is of the order of $100 \mu \mathrm{m}$, and is thus negligible, we are interested in the infinite permeability case $\lambda \rightarrow \infty$, which leads to the conditions

$$
u \geq \varphi, \quad \partial_{\nu} u \geq 0, \quad(u-\varphi) \partial_{\nu} u=0 \quad \text { on } \Gamma .
$$

Signorini called them ambiguous boundary conditions, but they are now known as Signorini boundary conditions. Since the solution $u$ is constrained to lie above $\varphi$ on $\Gamma$ (and not in the whole domain $\Omega$, as in the classical obstacle problem), $\varphi$ is referred to as the thin obstacle, and the problem is also called the thin obstacle problem (see Figure 8).

Fichera [Fic63] was the first one to establish existence and uniqueness in Signorini's problem by framing it in the setting of a Variational Inequality. With $\Omega, \Gamma$, and $\varphi$ as above, for a given function $g: \partial \Omega \rightarrow \mathbb{R}$ satisfying the compatibility condition $g>\varphi$ on $\Gamma$, the thin obstacle problem consists of solving the Variational Inequality

$$
\int_{\Omega} \nabla u \cdot \nabla(v-u) \geq 0 \quad \text { for every } v \in \mathcal{K},
$$

where

$$
\mathcal{K}=\left\{u \in W^{1,2}(\Omega): u=g \text { on } \partial \Omega \backslash \Gamma, u \geq \varphi \text { on } \Gamma\right\} .
$$

As in the classical obstacle problem, this is equivalent to minimizing the Dirichlet integral $J(v)=\int_{\Omega}|\nabla v|^{2}$ over $\mathcal{K}$.

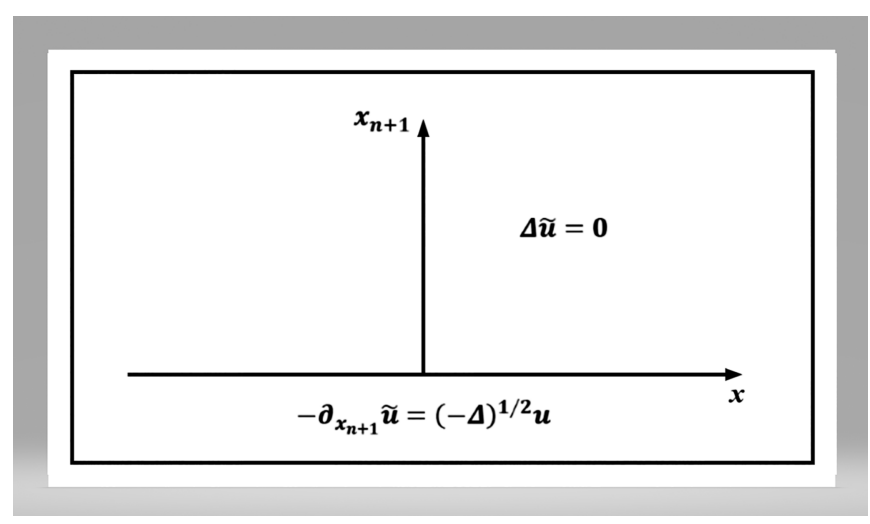

Figure 9. The Dirichlet-to-Neumann map.

An alternative interpretation of the thin obstacle problem is given as a "standard" obstacle problem as in (1), but with $-\Delta$ replaced by the fractional Laplacian

$$
(-\Delta)^{s} u(x)=C(n, s) \lim _{\varepsilon \rightarrow 0} \int_{\mathbb{R}^{n} \backslash B_{\varepsilon}(x)} \frac{u(x)-u(y)}{|x-y|^{n+2 s}} d y,
$$

where $0<s<1$ and $C(n, s)$ is a constant. The connection in the case $s=1 / 2$ comes through the Dirichlet-toNeumann map; see Figure 9. More precisely, let $u: \mathbb{R}^{n} \rightarrow$ $\mathbb{R}$ be a smooth function, and consider the extension problem

$$
\begin{array}{ll}
\Delta \tilde{u}=0 & \text { in } \mathbb{R}^{n} \times\left\{x_{n+1}>0\right\}, \\
\tilde{u}(x, 0)=u(x) & \text { for } x \in \mathbb{R}^{n},
\end{array}
$$

which yields a smooth bounded solution $\tilde{u}\left(x, x_{n+1}\right)$. Now replace $u$ with $-\partial_{n+1} \tilde{u}(x, 0)$ in the Dirichlet condition, to obtain $-\partial_{n+1} \tilde{u}\left(x, x_{n+1}\right)$ as the solution to the problem, instead of $\tilde{u}$. If we let

$$
T: u \mapsto-\partial_{n+1} \tilde{u}(x, 0),
$$

we thus have

$$
\begin{aligned}
T(T(u))(x) & =T\left(-\partial_{n+1} \tilde{u}(x, 0)\right)(x) \\
& =\partial_{n+1, n+1} \tilde{u}(x, 0)=-\Delta u(x) .
\end{aligned}
$$

It is easy to check that $T$ is a positive operator by an integration by parts argument. Therefore, we can conclude that $T=(-\Delta)^{1 / 2}$ and

$$
(-\Delta)^{1 / 2} u(x)=-\partial_{n+1} \tilde{u}(x, 0) .
$$

Hence, if $u$ is a solution of the obstacle problem for $(-\Delta)^{1 / 2}$ in $\mathbb{R}^{n}$ with smooth obstacle $\varphi$, then its harmonic extension $\tilde{u}$ to $\mathbb{R}^{n} \times(0,+\infty)$ solves the corresponding Signorini problem

$$
\begin{array}{ll}
\Delta \tilde{u}=0 & \text { in } \mathbb{R}^{n} \times \\
\tilde{u}(x, 0) \geq \varphi, \quad \partial_{n+1} \tilde{u}(x, 0) \leq 0 & \text { in } \mathbb{R}^{n}, \\
(\tilde{u}-\varphi) \partial_{n+1} \tilde{u}(x, 0)=0 & \text { in } \mathbb{R}^{n},
\end{array}
$$

and vice versa. With the two problems being equivalent, any regularity result for one of them can be carried over to the other one. There is a clear advantage, however, in using 
the Signorini type formulation, since it avoids the direct use of the nonlocal pseudodifferential operator $(-\Delta)^{1 / 2}$ by localizing the problem. This allows the use of purely local PDE methods, such as monotonicity formulas and classification of blow-up profiles, as we will discuss below.

One of the main reasons behind the resurgence of interest in nonlocal operators, for which the fractional Laplacian serves as a prototype, is that they are the infinitesimal generators of Markov processes relevant in financial mathematics. The infinitesimal generator of a discontinuous Markov process in $\mathbb{R}^{n}$ is no longer a differential operator, but rather an integro-differential, and therefore nonlocal, operator. It has been known for a long time that the infinitesimal generator of an isotropic $\alpha$-stable Lévy process is the fractional Laplacian operator of order $\alpha / 2(0<\alpha<2)$. More precisely, if $X(t)$ is such a process starting at zero and $x \in \mathbb{R}^{n}$, then

$$
(-\Delta)^{\alpha / 2} f(x)=\lim _{h \rightarrow 0^{+}} \frac{1}{h} \mathbb{E}[f(x)-f(x+X(h))] .
$$

Here, for a probability space $(S, \Sigma, P), f$ a bounded continuous function, and $X$ a random variable, $\mathbb{E}[f(X)]$ denotes the expected value of $f(X)$, that is,

$$
\mathbb{E}[f(X)]=\int_{S} f(X(\omega)) d P(\omega) .
$$

If we assume that $X(t)$ models the logarithm of an asset price and $\varphi: \mathbb{R}^{n} \rightarrow \mathbb{R}$ is the payoff function (i.e., a profit of $\varphi(s)$ is generated when trading the stock at $X(t)=s$ ), then one wants to maximize the expected profit

$$
u(x)=\sup \mathbb{E}[\varphi(X(\theta))],
$$

where the supremum is taken over all $0<\theta<\infty$, $\theta$ stopping time. The obstacle problem associated to nonlocal operators, such as the fractional Laplacian, arises in mathematical finance in the valuation of the so-called American option on multiple assets. An American option gives its holder the right to buy or sell a stock at a fixed price at any time prior to the set expiration time $T$. For instance, the S\&P 100 index option is an American option on a valueweighted index of 100 stocks. In the perpetual American option (that is, when $T \rightarrow \infty$ ), the value function $u$ is a solution to the obstacle-type problem

$$
\min \{L u, u-\varphi\}=0 \quad \text { in } \mathbb{R}^{n},
$$

where $L$ is the infinitesimal generator of $X(t)$. When the option has a finite expiration $T$, we note that the relevant operator $L$ is instead of parabolic type. If the stochastic process is Brownian motion, then $L=-\Delta$ and $u$ will satisfy the classical obstacle problem (1). However, modeling the behavior of stocks with possible discontinuities requires the use of jump processes, which have as infinitesimal generators integro-differential operators of the form

$$
L u(x)=\lim _{\varepsilon \rightarrow 0+} \int_{\mathbb{R}^{n} \backslash B_{\varepsilon}(x)}(u(y)-u(x)) K(x-y) d y .
$$

Such operators were introduced in finance by the Nobel Prize winner R. Merton.

In this context, the most important unknowns are the exercise region $\{u=\varphi\}$, in which one should exercise the option, and the continuation region $\{u>\varphi\}$, in which one instead should wait; see Figure 10. The free boundary is the interface separating the two. In the case of American put options, as in the case of American call options paying continuous dividends, it is always optimal to exercise early, and therefore the exercise region is nonempty. In general, solutions in this type of processes do not have closed form expressions, and thus it becomes important to determine the regularity of the boundary of this region, which in turn is closely related to the behavior of the value function for points on the free boundary.

Similarly to the classical obstacle problem, the main theoretical issues are the optimal regularity of the solution and the geometric analysis of the free boundary

$$
\mathcal{F}(u)=\partial\{x \in \Gamma \mid u(x)=\varphi(x)\} .
$$

The first result concerning the regularity of the solution in the Signorini problem is due to L. Caffarelli [Caf79], who proved that $u$ is $C^{1, \alpha}$ up to $\Gamma$ for some $0<\alpha \leq 1 / 2$, provided $\varphi \in C^{1,1}$. By analogy with the classical obstacle problem, however, one expects the regularity to be precisely $C^{1,1 / 2}$. This important fact was established in [AC04] for flat $\Gamma$ and vanishing obstacle $\varphi$. To get some intuition behind this result, we recall the observation (first made by H. Lewy) that in dimension $n=2$ the function

$$
u_{0}(x)=\rho^{3 / 2} \cos \left(\frac{3}{2} \theta\right)
$$

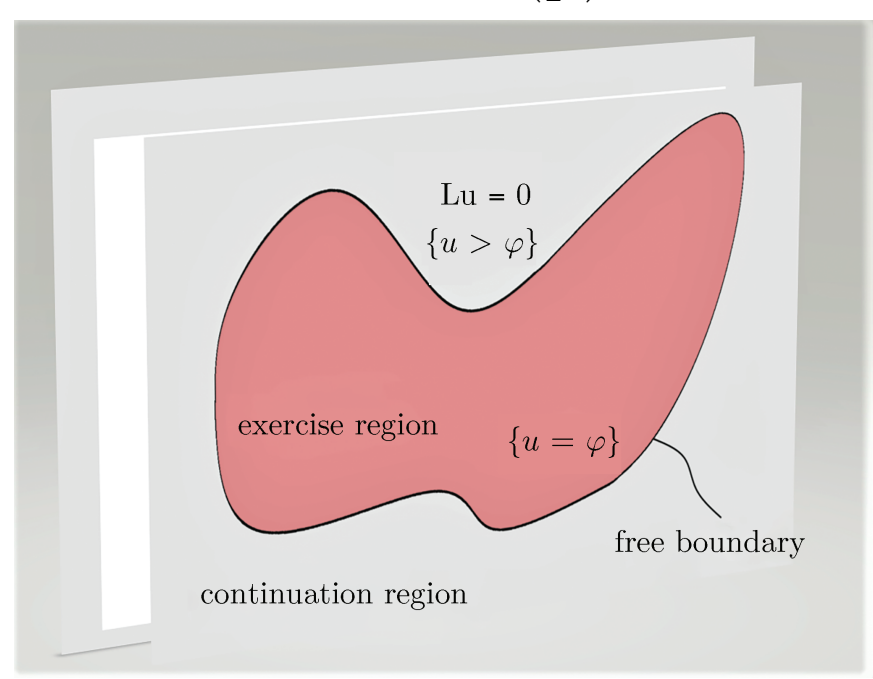

Figure 10. The free boundary separates the exercise region from the continuation region. 
is a homogeneous global solution to the Signorini problem. The main ingredients in the proof in [AC04] are a quasi-convexity property of the solution and a monotonicity formula for an appropriately weighted average of the local energy of the normal derivative of the solution.

This result opened the way to study regularity properties of the free boundary using geometric PDEs techniques. In [ACS08], I. Athanasopoulos, L. Caffarelli, and S. Salsa, under the same assumptions of flat boundary and zero obstacle, proved the existence of a basic global nondegenerate blow-up profile. Additionally, at points attaining such profile, they showed that the free boundary is a $C^{1, \alpha_{-}}$"curve," or, more precisely, a $C^{1, \alpha}(n-2)$-dimensional graph on the $(n-1)$-dimensional boundary. The groundbreaking idea in [ACS08] consists in the novel use of a classical result, Almgren's monotonicity formula, to control a family of rescalings tailor-made for the Signorini problem. In its original formulation [Alm79], Almgren's result reads as follows. For a function $u$ harmonic in $B_{1}(0)$ and $0<r<1$, define the quantities

$$
D(r ; u)=\int_{B_{r}(0)}|\nabla u|^{2} d x
$$

and

$$
H(r ; u)=\int_{\partial B_{r}(0)} u^{2} d H^{n-1},
$$

where $d H^{n-1}$ denotes the Hausdorff measure on $\partial B_{r}(0)$. Then the frequency functional

$$
N(r ; u)=\frac{r D(r ; u)}{H(r ; u)}
$$

is nondecreasing in $r$. The reason for the name is that, given the function $u_{k}(\rho, \theta)=a_{k} \rho^{k} \sin (k \theta)$ in $\mathbb{R}^{2}$, it is readily checked that $N(r)=k$. Remarkably, it was shown in [ACS08] that the same monotonicity property continues to hold when the function $u$ is a solution to the Signorini problem in

$$
\Omega=B_{1}^{+}:=B_{1}(0) \cap\left\{x_{n}>0\right\},
$$

with $\Gamma=B_{1}^{\prime}:=B_{1}(0) \cap\left\{x_{n}=0\right\}$ and $\varphi \equiv 0$ (assuming that $u$ is extended to the whole of $B_{1}(0)$ by even symmetry in $x_{n}$ ). An important-albeit immediate-consequence of this fact is that there exists

$$
N(0+; u):=\lim _{r \rightarrow 0^{+}} N(r ; u) .
$$

Another corollary, to keep in mind for future purposes, is a growth estimate of the solution at free boundary points: if $0 \in \mathcal{F}(u)$ and $\mu \leq N(0+; u)$, then there exists a positive constant $C$ such that

$$
\sup _{B_{r}}|u| \leq C r^{\mu} \quad \text { for } 0<r<\frac{1}{2} .
$$

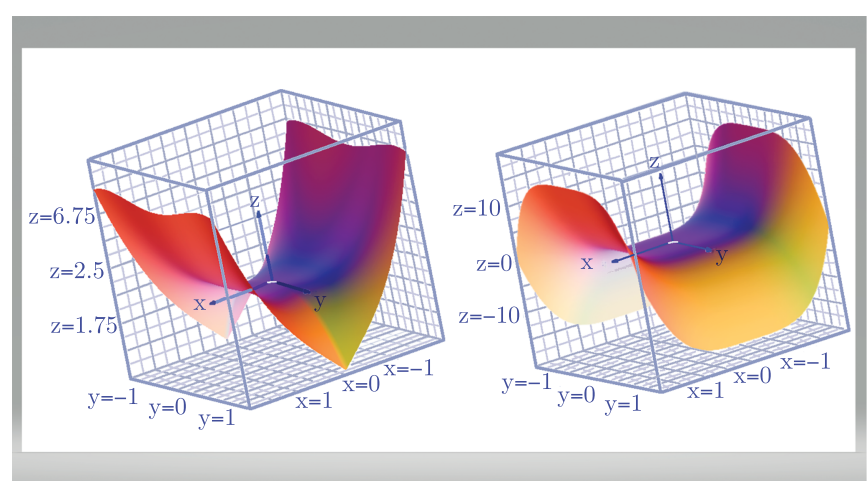

Figure 11. The graphs of $u_{0}(x)=\rho^{3 / 2} \cos \left(\frac{3}{2} \theta\right)$ (left) and $u_{0}(x)=\rho^{2} \cos (2 \theta)$ (right).

The next step in the program, following the roadmap provided by the classical obstacle problem, is the classification of free boundary points by analyzing the corresponding blow-ups. We introduce the family of Almgrenrescalings

$$
u_{r}(x)=\frac{u(r x)}{\left(r^{1-n} H(r ; u)\right)^{1 / 2}} .
$$

In order to apply compactness arguments to extract a convergent subsequence, it is necessary to control the $L^{2}$-norm of $\nabla u_{r}$, uniformly in $r>0$. To this end, we observe that

$$
\int_{B_{1}(0)}\left|\nabla u_{r}\right|^{2}=N\left(1, u_{r}\right)=N(r, u) \leq N(1, u),
$$

where we have used, in order, the definition of $N(r ; u)$, a simple change of variable, and the monotonicity of $N(r ; u)$ for $0<r \leq 1$. This inequality gives the desired control, and allows one to pass to the limit on a subsequence $r_{j} \rightarrow 0$ to obtain a blow-up profile $u_{0} \in W^{1,2}\left(B_{1}^{+}\right)$. Thanks to the $C^{1, \alpha}$ regularity of solutions, the convergence is actually in $C_{l o c}^{1}\left(B_{1}^{+} \cup B_{1}^{\prime}\right)$. This fact, in turn, coupled with the observation that $\left\|u_{r}\right\|_{L^{2}\left(\partial B_{1}\right)}=1$, allows one to prove that $u_{0}$ is a nonzero global solution of the Signorini problem (i.e., in $B_{R}^{+}$for all $R>0$ ). In addition, even if the blow-up may not be unique, depending on the choice of the subsequence chosen in the limiting process, it is characterized by the fact that it is homogeneous of degree $\mu:=N(0+; u)$. This process can be repeated at any free boundary point $x_{0} \in \mathcal{F}(u)$ by simply translating the point to the origin, that is, centering the balls in the definition of $N(r ; u)$ in $x_{0}$ instead of 0 . We will denote by $N\left(0+; u, x_{0}\right)$ the corresponding value.

The precise value of $N\left(0+; u, x_{0}\right)$ plays a preeminent role in the study of the structure of the free boundary. As a preliminary observation, from the optimal $C^{1,1 / 2}$-regularity of global solutions, one deduces that necessarily $\mu \geq 3 / 2$. In addition, it can be shown that if $3 / 2 \leq \mu<2$, then necessarily $\mu=3 / 2$ and up to a multiplicative constant and in a suitable system of coordinates, $u_{0}$ is in the form given 
in (6), with $\rho=x_{n-1}^{2}+x_{n}^{2}$ and $\tan \theta=x_{n} / x_{n-1}$ (see Figure 11). We will soon see that points of minimal frequency $\mu=3 / 2$ share many similarities with the regular points in the classical obstacle problem, and therefore we introduce the regular set as

$$
\mathcal{R}(u)=\left\{x_{0} \in \mathcal{F}(u) \mid N\left(0+; u, x_{0}\right)=3 / 2\right\} .
$$

To begin with, we note that the free boundary is locally Lipschitz continuous around regular points, i.e. (after possibly a rotation in $\mathbb{R}^{n-1}$ ),

$$
\mathcal{R}(u) \cap B_{\rho}^{\prime}=\left\{x_{n-1}=g\left(x^{\prime \prime}\right) \mid x \in B_{\rho}^{\prime \prime}\right\},
$$

with $g \in C^{0,1}\left(B_{\rho}^{\prime \prime}\right)$. Here $x^{\prime \prime}=\left(x_{1}, \ldots, x_{n-2}\right)$, and $B_{\rho}^{\prime \prime}$ is the ball in $\mathbb{R}^{n-2}$ centered at the origin and of radius $\rho$. The idea of the proof is analogous to the one for the classical obstacle problem, and it relies on the explicit knowledge of the blow-up limit and the existence of a thin cone of directions

$$
C_{\delta}^{\prime}=\left\{x=\left(x^{\prime \prime}, x_{n-1}\right) \in \mathbb{R}^{n-1}\left|x_{n-1}>\delta\right| x^{\prime \prime} \mid\right\}
$$

along which the solution is monotone.

To establish further regularity properties, the following result, known as the Boundary Harnack Principle, is crucial. It states that the quotient of two positive harmonic functions $u_{1}$ and $u_{2}$ in a Lipschitz domain $\Omega$, both vanishing on $\partial \Omega$, satisfies the following Harnack-type inequality:

$$
\sup _{B_{R} \cap \Omega} \frac{u_{1}}{u_{2}} \leq C \inf _{B_{R} \cap \Omega} \frac{u_{1}}{u_{2}} .
$$

From this follows, in a rather standard fashion, the Hölder continuity of the quotient itself:

$$
\underset{B_{\rho} \cap \Omega}{\operatorname{osc}} \frac{u_{1}}{u_{2}} \leq C\left(\frac{\rho}{r}\right)^{\alpha} \underset{B_{r} \cap \Omega}{\operatorname{osc}} \frac{u_{1}}{u_{2}}
$$

for $0<\rho<r$. We now note that $\partial_{e} u$ is nonnegative in a small ball $B_{r_{\delta}}(0)$ for any unit vector $e \in C_{\delta}^{\prime}$, and it is harmonic in $B_{r_{\delta}}(0) \backslash\{u=0\}$. One would like to apply the Boundary Harnack Principle to the ratio $\partial_{e} u / \partial_{e_{n-1}} u$ to infer that it is of class $C^{\alpha}$ in a small ball $B_{\rho}(0)$. However, this is not immediately possible since the domain $B_{r_{\delta}}(0) \backslash\{u=0\}$ is not quite Lipschitz, being lower-dimensional. It is possible, though, to transform it into one by means of a biLipschitz transformation $T$ that maps $B_{r_{\delta}}(0) \backslash\{u=0\}$ onto, say, the upper half-ball $\left\{|z|<1, z_{n-1}>0\right\}$. This mapping will transform the Laplacian into a uniformly elliptic operator in divergence form. Applying an appropriate version of the Boundary Harnack Principle for this class of equations first, and the inverse transformation $T^{-1}$ afterwards, gives the desired Hölder continuity of $\partial_{e} u / \partial_{e_{n-1}} u$. From this it is easy to deduce that

$$
\frac{\partial_{e_{i}} u}{\partial_{e_{n-1}} u} \in C^{\alpha}\left(B_{\rho}(0)\right), \quad i=1, \ldots, n-2 .
$$

At this point it suffices to observe that if $g$ is as in (9), then (at least heuristically)

$$
\partial_{e_{i}} g=\frac{\partial_{e_{i}} u}{\partial_{e_{n-1}} u}, \quad i=1, \ldots, n-2 .
$$

Hence, we have shown that the regular part of the free boundary $\mathcal{R}(u)$ is a locally $C^{1, \alpha}(n-2)$-dimensional graph, as stated above. It is important to note that this result (together with the optimal regularity of solutions) has been generalized to nonzero obstacles $\varphi \in C^{2,1}$ and to all fractional Laplacians $(-\Delta)^{s}$ for $0<s<1$ by L. Caffarelli, S. Salsa, and L. Silvestre in [CSS08]. Their approach is based on a PDE realization of $(-\Delta)^{s}$ developed by Caffarelli and Silvestre in [CS07], which generalizes the Dirichlet-to-Neumann map for the case $s=1 / 2$. Indeed, in a weak sense it holds that

$$
(-\Delta)^{s} u(x)=-\kappa_{a} \lim _{x_{n+1} \rightarrow 0^{+}} x_{n+1}^{a} \partial_{n+1} \tilde{u}\left(x, x_{n+1}\right)
$$

for a suitable constant $\kappa_{a}, a=1-2 s$, and $\tilde{u}$ solution to the problem

$$
\begin{array}{ll}
\mathcal{L}_{a} \tilde{u}=\operatorname{div}\left(x_{n+1}^{a} \nabla \tilde{u}\right)=0 & \text { in } \mathbb{R}^{n} \times(0,+\infty), \\
\tilde{u}(x, 0)=u(x) & \text { in } \mathbb{R}^{n} .
\end{array}
$$

This extension procedure allows one to transform the obstacle problem for $(-\Delta)^{S}$ into a Signorini-type problem for the degenerate operator $\mathcal{L}_{a}$, which can be studied with proper generalizations of the techniques we have just described.

More recently, higher regularity properties of $\mathcal{R}(u)$ have been proved in [KPS15] and [DSS16]. Specifically, in the former paper $\mathrm{H}$. Koch, A. Petrosyan, and W. Shi show that the regular part of the free boundary is real analytic by using a partial hodograph-Legendre transformation, whereas in the latter D. De Silva and O. Savin obtain the $C^{\infty}$ regularity of $\mathcal{R}(u)$ by means of a higher-order boundary Harnack inequality.

This shows that regular points are aptly named, as it so happens in the classical obstacle problem. A substantial difference from the latter, however, rests in the fact that there is no dichotomy in the subclassification of $\mathcal{F}(u)$. Already in the 2-dimensional case, in fact, one observes that the Signorini problem admits global homogeneous solutions of the form $\rho^{\kappa} \cos (\kappa \theta)$ for $\kappa=2 m$ or $\kappa=2 m+1 / 2$, with $m \in \mathbb{N}$ (see Figure 11). Proceeding by analogy with the classical theory, we now focus our attention on the free boundary points at which the contact set has vanishing density. We define the singular set $\Sigma(u)$ as the collection of points $x_{0} \in \mathcal{F}(u)$ such that

$$
\limsup _{r \rightarrow 0^{+}} \frac{\mathcal{H}^{n-1}\left(B_{r}^{\prime}\left(x_{0}\right) \cap\{u=0\}\right)}{r^{n-1}}=0 .
$$

Following the ideas introduced by N. Garofalo and A. Petrosyan in [GP09], we shall see that $\Sigma(u)$ is contained in the 
union of $C^{1}$-manifolds of suitable dimension. Before doing so, though, we observe that there are situations where the entire free boundary could be composed by singular points, as the example

$$
p\left(x_{1}, x_{2}, x_{3}\right)=x_{1}^{2} x_{2}^{2}-\left(x_{1}^{2}+x_{2}^{2}\right) x_{3}^{2}+\frac{1}{3} x_{3}^{4}
$$

shows. This is, in fact, a solution of the zero-obstacle Signorini problem in $B_{1}^{+}$, with $\mathcal{F}(u)=\{p=0\}$ given by the union of the lines

$$
x_{1}=x_{3}=0 \text { and } x_{2}=x_{3}=0 .
$$

The starting point is a fundamental characterization of singular points, given by the three following equivalent properties:

(i) $x_{0} \in \Sigma(u)$.

(ii) Any blow-up limit of $u$ at $x_{0}$ is a nonnegative, nonidentically zero polynomial, homogeneous of degree $\mu$, harmonic, and even in $x_{n}$. We will denote by $\mathcal{P}_{\mu}$ the class of such polynomials.

(iii) $\mu=2 m, m \in \mathbb{N}$.

Given the prominent role occupied by the homogeneity of the blow-ups in describing the structure of the free boundary, it becomes natural to classify points in $\Sigma(u)$ accordingly. We thus let

$$
\Sigma_{\mu}(u)=\left\{x \in \mathcal{F}(u) \mid N\left(0+; u, x_{0}\right)=\mu\right\} .
$$

Next, following the blueprint provided by the classical obstacle problem, we introduce two one-parameter families of monotonicity formulas. The first result is for functionals of Weiss-type, and it consists in the nondecreasing character, for $0<r<1$, of the functional

$$
\begin{aligned}
\mathcal{W}_{\mu}(r ; u)= & \frac{1}{r^{n-2+2 \mu}} \int_{B_{r}}|\nabla u|^{2} \\
& -\frac{\mu}{r^{n-1+2 \mu}} \int_{\partial B_{r}} u^{2} .
\end{aligned}
$$

It should be noted that Weiss's functional is closely related to Almgren's frequency function, as they are both constructed with the same building blocks $D(u)=\int_{B_{r}}|\nabla u|^{2}$ and $H(r)=\int_{\partial B_{r}} u^{2}$. The second family of functionals is of Monneau-type, given by

$$
\mathcal{M}_{\mu}(r ; u, p)=\frac{\mu}{r^{n}-1+2 \mu} \int_{\partial B_{r}}(u-p)^{2} .
$$

If $0 \in \Sigma_{\mu}$ and $p \in \mathcal{P}_{\mu}(\mu=2 m$ for some $m \in \mathbb{N})$, then $\mathcal{M}_{\mu}(r ; u, p)$ is nondecreasing for $0<r<1$. A first consequence of this monotonicity formula is a nondegeneracy property for solutions at singular points $x_{0} \in \Sigma_{\mu}$, i.e.,

$$
\sup _{\partial B_{r}\left(x_{0}\right)}|u(x)| \geq r^{\mu} \text {. }
$$

In order to proceed, we should recall that the power of monotonicity formulas lies in the fact that they allow one to control a suitable family of rescaled functions. For regular points, we have seen that Almgren's frequency function works in pair with the namesake family of rescalings defined in (8). Said family, however, is not well suited to describe the behavior of the free boundary at singular points, nor is it fitting to be paired with Monneau's monotonicity property. We turn, once more, to the classical obstacle problem for inspiration, in particular to (2) and (3). Observing that, if $x_{0} \in \Sigma_{\mu}$, it follows from (7) and (10) that $u(x) \simeq r^{\mu}$ in $B_{r}\left(x_{0}\right)$, the logical choice of rescalings is given by

$$
u_{r}^{(\mu)}(x)=\frac{u\left(x_{0}+r x\right)}{r^{\mu}} .
$$

With this notion at hand, it is possible to proceed as illustrated in the previous section to prove a structural theorem for $\Sigma_{\mu}(u)$. Namely, for $x_{0} \in \Sigma_{\mu}$ and $p \in \mathcal{P}_{\mu}$ as in (ii) above, define the dimension of $\Sigma_{\mu}$ at $x_{0}$ as

$d_{x_{0}}^{\mu}=\operatorname{dim}\left\{\xi \in \mathbb{R}^{n-1} \mid \xi \cdot \nabla_{x^{\prime}} p\left(x^{\prime}, 0\right)=0\right.$ for all $\left.x^{\prime} \in \mathbb{R}^{n-1}\right\}$.

Next, for $d=0,1, \ldots, n-2$, let

$$
\Sigma_{\mu}^{d}=\left\{x_{0} \in \Sigma_{\mu} \mid d_{x_{0}}^{\mu}=d\right\} .
$$

Then, every set $\Sigma_{\mu}^{d}$, with $\mu=2 m(m \in \mathbb{N})$ and $d=0, \ldots, n-$ 2 , is contained in a countable union of $d$-dimensional $C^{1}$ manifolds. This result has been very recently improved upon in [CSV20], where M. Colombo, L. Spolaor, and B. Velichkov prove an explicit logarithmic modulus of continuity.

\section{Concluding Remarks}

The presentation in this article focused only on two basic models of obstacle problems. Its purpose is only to give a bird's-eye view of the topic, and of the new mathematical tools and ideas whose developments it has fostered. Many generalizations to other classes of operators (quasi-linear and fully nonlinear, with variable coefficients, integrodifferential, and time-dependent, just to name a few) have been extensively studied over the years, and continue to be developed. Due to the restriction on the number of references, the author could not acknowledge several important contributions, both old and new. For instance, a more refined analysis of the structure and regularity of the free boundary in the Signorini problem, correlated with the study of admissible frequencies, has been the object of active investigation as of late. This has been made possible by the introduction of delicate epiperimetric inequalities, modeled on the one for the classical obstacle problem established by Weiss [Wei99]. For a more comprehensive treatment of some of these aspects, the author refers to the monograph [PSU12] and to the survey article [DS18], and to the references therein. 
ACKNOWLEDGMENTS. This article is dedicated to Luis Caffarelli, whose beautiful and groundbreaking ideas have been inspiring generations of mathematicians. The author is grateful to Nicola Garofalo, Sandro Salsa, and the reviewers for helpful conversations and comments.

\section{References}

[AC04] I. Athanasopoulos and L. A. Caffarelli, Optimal regularity of lower dimensional obstacle problems (English, with English and Russian summaries), Zap. Nauchn. Sem. S.Peterburg. Otdel. Mat. Inst. Steklov. (POMI) 310 (2004), no. Kraev. Zadachi Mat. Fiz. i Smezh. Vopr. Teor. Funkts. 35 [34], 49-66, 226, DOI 10.1007/s10958-005-0496-1 English transl., J. Math. Sci. (N.Y.) 132 (2006), no. 3, 274-284. MR2120184

[ACS08] I. Athanasopoulos, L. A. Caffarelli, and S. Salsa, The structure of the free boundary for lower dimensional obstacle problems, Amer. J. Math. 130 (2008), no. 2, 485-498. MR2405165

[Alm79] Frederick J. Almgren Jr., Dirichlet's problem for multiple valued functions and the regularity of mass minimizing integral currents, Minimal submanifolds and geodesics (Proc. Japan-United States Sem., Tokyo, 1977), North-Holland, Amsterdam-New York, 1979, pp. 1-6. MR574247

[Caf77] Luis A. Caffarelli, The regularity of free boundaries in higher dimensions, Acta Math. 139 (1977), no. 3-4, 155184. MR454350

[Caf79] L. A. Caffarelli, Further regularity for the Signorini problem, Comm. Partial Differential Equations 4 (1979), no. 9, 1067-1075, DOI 10.1080/03605307908820119 MR542512

[Caf98] L. A. Caffarelli, The obstacle problem revisited, J. Fourier Anal. Appl. 4 (1998), no. 4-5, 383-402, DOI 10.1007/BF02498216 MR1658612

[CS07] Luis Caffarelli and Luis Silvestre, An extension problem related to the fractional Laplacian, Comm. Partial Differential Equations 32 (2007), no. 7-9, 1245-1260, DOI 10.1080/03605300600987306. MR2354493

[CSS08] Luis A. Caffarelli, Sandro Salsa, and Luis Silvestre, Regularity estimates for the solution and the free boundary of the obstacle problem for the fractional Laplacian, Invent. Math. 171 (2008), no. 2, 425-461, DOI 10.1007/s00222007-0086-6 MR2367025

[CSV20] Maria Colombo, Luca Spolaor, and Bozhidar Velichkov, Direct epiperimetric inequalities for the thin obstacle problem and applications, Comm. Pure Appl. Math. 73 (2020), no. 2, 384-420, DOI 10.1002/cpa.21859. MR4054360

[DL76] G. Duvaut and J.-L. Lions, Inequalities in mechanics and physics, Grundlehren der Mathematischen Wissenschaften, vol. 219, Springer-Verlag, Berlin-New York, 1976. Translated from the French by C. W. John. MR0521262

[DS18] Donatella Danielli and Sandro Salsa, Obstacle problems involving the fractional Laplacian, Recent developments in nonlocal theory, 2018, pp. 81-164. MR3824211
[DSS16] Daniela De Silva and Ovidiu Savin, Boundary Harnack estimates in slit domains and applications to thin free boundary problems, Rev. Mat. Iberoam. 32 (2016), no. 3, 891-912, DOI 10.4171/RMI/902. MR3556055

[Fic63] Gaetano Fichera, Sul problema elastostatico di Signorini con ambigue condizioni al contorno (Italian), Atti Accad. Naz. Lincei Rend. Cl. Sci. Fis. Mat. Nat. (8) 34 (1963), 138-142. MR176661

[Fre72] Jens Frehse, On the regularity of the solution of a second order variational inequality (English, with Italian summary), Boll. Un. Mat. Ital. (4) 6 (1972), 312-315. MR0318650

[FS19] Alessio Figalli and Joaquim Serra, On the fine structure of the free boundary for the classical obstacle problem, Invent. Math. 215 (2019), no. 1, 311-366. MR3904453

[GP09] Nicola Garofalo and Arshak Petrosyan, Some new monotonicity formulas and the singular set in the lower dimensional obstacle problem, Invent. Math. 177 (2009), no. 2, 415-461, DOI 10.1007/s00222-009-0188-4 MR2511747

[KN77] D. Kinderlehrer and L. Nirenberg, Regularity in free boundary problems, Ann. Scuola Norm. Sup. Pisa Cl. Sci. (4) 4 (1977), no. 2, 373-391. MR440187

[KPS15] Herbert Koch, Arshak Petrosyan, and Wenhui Shi, Higher regularity of the free boundary in the elliptic Signorini problem, Nonlinear Anal. 126 (2015), 3-44, DOI 10.1016/j.na.2015.01.007. MR3388870

[Mon03] R. Monneau, On the number of singularities for the obstacle problem in two dimensions, J. Geom. Anal. 13 (2003), no. 2, 359-389, DOI 10.1007/BF02930701. MR1967031

[PSU12] Arshak Petrosyan, Henrik Shahgholian, and Nina Uraltseva, Regularity of free boundaries in obstacle-type problems, Graduate Studies in Mathematics, vol. 136, American Mathematical Society, Providence, RI, 2012. MR2962060

[Sig59] A. Signorini, Questioni di elasticità non linearizzata e semilinearizzata (Italian), Rend. Mat. e Appl. (5) 18 (1959), 95-139. MR118021

[SY19] O. Savin and H. Yu, Regularity of the singular set in the fully nonlinear obstacle problem, arXiv:1905.02308, 2019.

[Wei99] Georg S. Weiss, A homogeneity improvement approach to the obstacle problem, Invent. Math. 138 (1999), no. 1, 2350, DOI 10.1007/s002220050340 MR1714335

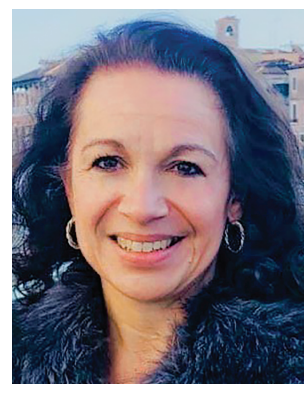

Donatella Danielli

Credits

All article images are courtesy of Donatella Danielli. Photo of Donatella Danielli is courtesy of Nicola Garofalo. 